\title{
Longitudinal volume changes of hippocampal subfields and cognitive decline in Parkinson's disease
}

\author{
Rui Xu ${ }^{1 \#}$, Xiaofei Hu${ }^{2 \#}$, Xiaomei Jiang ${ }^{3}$, Yanling Zhang ${ }^{2}$, Jian Wang ${ }^{2}$, Xianchun Zeng' \\ ${ }^{1}$ Department of Radiology, Guizhou Provincial People's Hospital, Guiyang 550002, China; ${ }^{2}$ Department of Radiology, Southwest Hospital, Third \\ Military Medical University (Army Medical University), Chongqing 400038, China; ${ }^{3}$ Department of Centre for Disease Prevention and Control, \\ Chengdu Military Region, Chengdu 610011, China \\ "These authors contributed equally to this work. \\ Correspondence to: Jian Wang. Department of Radiology, Southwest Hospital, Third Military Medical University, Chongqing 400038, China. \\ Email: wangjian_811@yahoo.com; Xianchun Zeng. Department of Radiology, Guizhou Provincial People’s Hospital, Guiyang 550002, China. \\ Email: zengxianchun04@foxmail.com.
}

Background: Neuropathological studies have shown that the hippocampus is affected in Parkinson's disease (PD) with cognitive impairment. Our goal was to assess the longitudinal volume change of different hippocampal subfields in PD patients with and without cognitive decline using magnetic resonance imaging (MRI).

Methods: A total of 28 nondemented PD patients and 27 neurologically unimpaired elderly controls were enrolled in this study, and three-dimensional (3D) T1-weighted MRI was performed. All PD patients that were followed up and rescanned after 2 years were divided into two groups: PD without cognitive decline $(n=15)$ and PD with cognitive decline $(n=13)$. A Bayesian model implemented in FreeSurfer was used to segment the hippocampal subfields automatically. Scale for global cognitive status included the Mini Mental State Examination (MMSE) and the Montreal Cognitive Assessment (MoCA).

Results: In the cross sectional study, the bilateral hippocampal volume was smaller in PD patients compared to healthy controls, and the bilateral subiculum, CA2/3, CA4, and molecular layer (ML) subfields, and the right granule cell layer of the dentate gyrus (GC-DG) subfield, were significantly decreased in the PD patients. Significant correlations were found between the MoCA score and total hippocampus volume in PD patients. In the follow-up group, bilateral CA4, ML, and GC-DG subfields, and left CA2/3 and right presubiculum subfields, were significantly smaller in PD patients with cognitive decline compared to PD patients without cognitive decline. Significant correlations were found between the longitudinal change of the MMSE or MoCA scores and percent change rate of total bilateral hippocampal, bilateral ML, and right CA4 in all PD patients.

Conclusions: Our results demonstrated the selective regional vulnerability of the hippocampus in the progression of PD. These findings corroborate neuropathological findings and add novel information about the involvement of the hippocampus in the cognitive dysfunction of PD.

Keywords: Parkinson's disease (PD); hippocampal subfields; magnetic resonance imaging (MRI); cognitive decline; longitudinal study

Submitted May 19, 2019. Accepted for publication Oct 21, 2019.

doi: $10.21037 /$ qims.2019.10.17

View this article at: http://dx.doi.org/10.21037/qims.2019.10.17 


\section{Introduction}

Cognitive impairment is one of the most common nonmotor symptoms in Parkinson's disease (PD), even early in the disease course $(1,2)$. Studies have described impairments in different cognitive subdomains in newly diagnosed PD patients $(3,4)$. Furthermore, longitudinal assessments of cognition have revealed a faster rate of cognitive decline in these early PD patients than in healthy people $(5,6)$.

Decreases in cognitive functioning have been found related to hippocampal atrophy in $\mathrm{PD}$, but the findings are inconsistent (7-9). One pathological study showed that hippocampal changes were present in the relatively early stages of PD patients (10), and these changes were also found to occur in both demented and non-demented patients (11). However, the majority of previous studies compared total hippocampal volumes using a regionof-interest approach, without evaluating the various hippocampal subregions, such as cornus ammonis 1 (CA1), CA2-3, CA4, dentate gyrus (DG), along with the subiculum and presubiculum.

A pathological study has demonstrated Lewy neurites or $\alpha$-synuclein stack in the hippocampus of PD patients, especially in the CA2/3 and CA4-DG subfields $(10,12)$. Compared with non-demented patients, PD patients with dementia have higher densities of Lewy bodies and Lewy neuritis in the CA2/3 area. Moreover, there is a correlation between the density of Lewy neurites in hippocampal area CA2 and the severity of cognitive impairment (11). Considering the functional specialization of the histologically distinct subfields of the hippocampus and the results of the histopathological studies described above, further study of the hippocampal subfield changes in PD, especially longitudinal study, is necessary.

Our goal was to investigate the feature of hippocampal subfields volumetry change in PD, especially longitudinal subfield changes and their role in cognitive decline. Based on previous studies, we hypothesized that PD with cognitive decline would exhibit distinctive patterns of volumetry change. We also hypothesized that the CA2/3 and CA4-DG region would be the most vulnerable subfields and would be related to cognitive decline in $\mathrm{PD}$ patients.

\section{Methods}

\section{Subjects}

The present study was approved by the Ethics Committee of Southwest Hospital, and written informed consent to participate in the study was obtained from all subjects. Twenty-eight nondemented PD patients from the movement disorders outpatient clinic were included. In addition, 27 age- and gender-matched healthy controls were recruited. All of the subjects were right-handed, as measured by the Edinburgh Handedness Inventory (13). The inclusion criteria for patients included idiopathic PD as defined using the UK Brain Bank criteria (14). The exclusion criteria included the following: presence of other neurological disorders aside from PD; meeting the diagnostic criteria for PD dementia (15); parkinsonism due to antipsychotics or other drugs; pre-existing neurological or psychiatric disorders; use of alcoholism or drug abuse; and incidental problems (stroke, white matter disease) and brain lesion or injury on MRI.

The patients were assessed by a trained physician with the Unified Parkinson's Disease Rating Scale (UPDRS)III motor scale (16) and the Hoehn and Yahr scale (17). The assessments were conducted during an off state after the administration of anti-parkinsonian drugs was withheld for at least $12 \mathrm{~h}$ overnight. Only mild-to-moderate stage patients were enrolled in the study to ensure the long scan could be completed. The scales for global cognitive status included the Mini Mental State Examination (MMSE) and Montreal Cognitive Assessment (MoCA). Twenty-eight PD patients were rescanned after two years (mean 899 days, SD 36), and these patients were assessed with the UPDRSIII motor scale, Hoehn scale, Yahr scale, MMSE, and MoCA by the same physician. Studies have suggested that the MoCA may be more sensitive to cognitive impairment for detecting longitudinal changes and at baseline in PD $(18,19)$. Thus, we defined the global cognitive decline subgroup based on the following criteria: (I) PD patients with decreased scores of only MoCA at least or both MoCA and MMSE; (II) emerging subjective cognitive problems reported by patients or family members. Fifteen patients were classified as having global cognitive decline, and 13 patients were classified as without global cognitive decline. All participants were right-handed, as assessed by the Edinburgh Handedness Inventory (13).

\section{Data acquisition}

The images were acquired with a 3.0-T Siemens Tim Trio whole-body magnetic resonance imaging (MRI) system (Siemens Medical Solutions, Erlangen, Germany). Threedimensional (3D) T1-weighted anatomical images were acquired in a sagittal orientation using the following 
volumetric 3D magnetization-prepared rapid gradientecho (MP-RAGE) sequence $(\mathrm{TR}=1,900 \mathrm{~ms}, \mathrm{TE}=2.52 \mathrm{~ms}$, flip angle $=9^{\circ}$, slice thickness $=1 \mathrm{~mm}$, slices $=176$, field of view $=256 \times 256 \mathrm{~mm}^{2}$, matrix size $=256 \times 256$, voxel size $=1 \times 1 \times 1 \mathrm{~mm}^{3}$ ) on each subject.

\section{Image processing}

Hippocampus segmentation was performed with the FreeSurfer software (version 6.0) (http://surfer.nmr.mgh. harvard.edu/). Information on the procedure details is freely available for download online (http://www.freesurfer.net/ fswiki/LongitudinalHippocampalSubfields). Detailed steps of hippocampal subfield segmentation have been described in previous studies $(20,21)$. The hippocampal subfields the FreeSurfer segmented included the parasubiculum, presubiculum, subiculum, CA1, CA2/3, CA4, the granule cell layer of the DG (GC-DG), the molecular layer (ML), fimbria, the hippocampal fissure, the hippo-campusamygdala transition area (HATA), and the hippocampal tail (the posterior end of the hippocampus, which includes portions of the CA fields and DG undistinguishable with the MRI contrast). The intracranial volume (ICV) of each subject was also estimated.

To measure volume changes of the total hippocampus and hippocampal subfields over time, FreeSurfer's longitudinal stream was used for longitudinal analyses, which creates an unbiased within-subject template space and image using robust inverse consistent registration (21). The procedure uses a computational atlas built from high-resolution ex vivo MRI data, acquired at an average of $0.13 \mathrm{~mm}$ isotropic resolution on a 7-T scanner, and an in vivo atlas that provides information about adjacent extrahippocampal structures (22). The unbiased longitudinal segmentation relies on subjectspecific atlases, and the segmentation at the different time points are jointly computed using a Bayesian inference algorithm (21).

Volumes of the hippocampus and hippocampal subfield were adjusted by total intracranial volume (TIV): volume $_{(\text {adjusted) }}=$ volume $_{(\text {observed })} \times \mathrm{TIV}_{\mathrm{m}} / \mathrm{TIV}_{\mathrm{i}}$

Where volume $_{\text {(adjusted) }}$ is the normalized volume of a subject, volume $_{\text {(observed) }}$ is the non-normalized volume, $\mathrm{TIV}_{\mathrm{m}}$ is the mean TIV of all subjects, and $\mathrm{TIV}_{\mathrm{i}}$ is individual TIV of the subject (23).

\section{Statistical analyses}

The distribution of continuous variables was tested using the Skewness-Kurtosis test. Demographic differences between the PD group and healthy control at baseline, and between the two PD groups (PD without cognitive decline group $v s$. PD with cognitive decline group) at baseline were assessed with t-tests or Mann-Whitney tests depending on the normality of distributions. The Chi-squared test was used to examine sex distributions between the groups. The paired Wilcoxon signed-rank test was used to compare differences in neuropsychological variables over time (baseline and follow-up surveys). Longitudinal changes on the MoCA and MMSE total scores were determined using repeated-measures analysis of variance (ANOVA) (time $x$ group). $[\mathrm{P}<0.05$, corrected with false discovery rate (FDR)].

Comparisons of hippocampal subfield volumes between the PD group and healthy control at baseline were examined with analysis of covariance (ANCOVA), controlling for age, sex, education, and ICV. Longitudinal changes in hippocampal subfield volume were also determined using repeated measures ANOVA (mixed-effects model) with group as a between-subjects factor (PD without cognitive decline group vs. PD with cognitive decline group), and time (baseline, follow-up) as a within-subjects repeated measure, with age as a covariate $(23,24)(\mathrm{P}<0.05$, corrected with FDR).

Within each group, we further investigated the correlation analyses between the MMSE and MoCA scores and total hippocampal or subfield volumes, and the longitudinal change of the MMSE or MoCA scores and mean percent change rate of total hippocampal or subfield volumes between two scan and mean percent change rate, which was calculated by the following formula: (volume baseline - volume $_{\text {follow-up }}$ ) $\times 100 \% /$ volume $_{\text {baseline }}(24)$.

\section{Results}

\section{Demographic data and hippocampal subfield volumes at baseline}

There were no differences in age, gender, education, MMSE, and MoCA total scores between the PD and control groups (Table 1).

ANCOVA showed that total bilateral hippocampal volume was significantly decreased in the PD patients compared to the controls (Table 2, Figure 1) (left: $\mathrm{P}<0.001$, right: $\mathrm{P}=0.012$ ). The hippocampal subfields showed distinct patterns of between-group differences (Table 2, Figure 1). The bilateral CA2/3 (left: $\mathrm{P}=0.025$, right: $\mathrm{P}=0.018$ ), CA4 (left: $\mathrm{P}<0.001$, right: $\mathrm{P}<0.001$ ), $\mathrm{ML}$ (left: $\mathrm{P}<0.001$, right: 
Table 1 Demographic information and clinical characteristics of baseline subjects

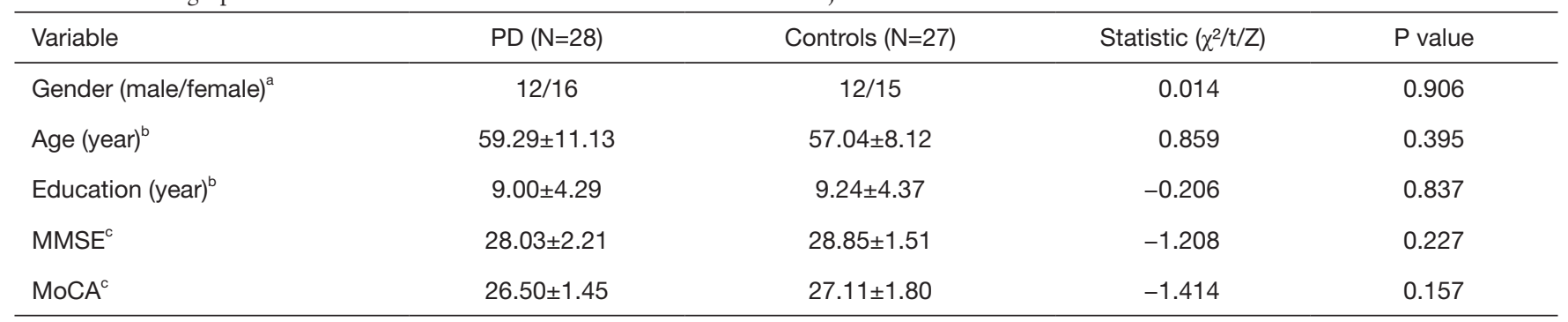

${ }^{a}$, the $\mathrm{P}$ value for gender distribution in the two groups was calculated using chi-squared test. ${ }^{\mathrm{b}}$, the $\mathrm{P}$ value was calculate using two-tail two-sample $t$-test. ${ }^{c}$, the P value was calculate using two-tail Mann-Whitney U test. MMSE, Mini Mental State Examination; MoCA, Montreal Cognitive Assessment; PD, Parkinson's disease.

$\mathrm{P}=0.003$ ), subiculum (left: $\mathrm{P}<0.001$, right: $\mathrm{P}<0.001$ ), and right $\mathrm{GC}-\mathrm{DG}(\mathrm{P}<0.001)$ regions were smaller in the $\mathrm{PD}$ patients than those in the controls. The correlation analyses showed that MoCA total score correlated with bilateral total hippocampal volume in the PD group (Figure 1).

\section{Changes in clinical data and hippocampal subfield volumes for the longitudinal PD cohort}

There were no differences in age, gender, education, disease duration, UPDRS-III score, MMSE, and MoCA total scores between PD without cognitive decline group and PD with cognitive decline group in baseline (Table 3). However, paired Wilcoxon signed-rank test showed that there were significant differences in MMSE $(\mathrm{P}=0.003)$ and MoCA $(\mathrm{P}=0.014)$ scores between baseline and follow-up in the two groups, and repeated measures ANOVA showed that score of MMSE $(\mathrm{P}<0.001)$ and MoCA $(\mathrm{P}=0.001)$ was significantly decreased in the $\mathrm{PD}$ with cognitive decline group (Tables 4,5).

The differences in hippocampal subfields between groups had distinctive patterns. The volume of total bilateral hippocampal (left: $\mathrm{P}=0.016$, right: $\mathrm{P}=0.004$ ), bilateral CA4 (left: $\mathrm{P}=0.007$, right: $\mathrm{P}=0.011$ ), GC-DG (left: $\mathrm{P}=0.003$, right: $\mathrm{P}=0.016$ ), $M L$ (left: $\mathrm{P}=0.011$, right: $\mathrm{P}=0.014$ ), and left $\mathrm{CA} 2 / 3(\mathrm{P}=0.002)$ and right presubiculum $(\mathrm{P}=0.002)$ showed a statistically significant group-by-time interaction, and the atrophy rate of the total bilateral hippocampal and above subfield was faster in PD patients with cognitive decline (Table 6, Figure 2). Significant correlations were found between the longitudinal change of the MMSE and MoCA scores which had significant positive correlations with the mean percent change rate of the total bilateral hippocampal and subfield volumes (GC-DG, ML and CA4) in all PD patients (PD without cognitive decline group and $\mathrm{PD}$ with cognitive decline group) (Figures 3,4).

\section{Discussion}

This study explored the pattern of decrease in the volume of the total hippocampus and hippocampal subfields in PD patients. In the baseline groups, the total bilateral hippocampal volume, bilateral subiculum, CA2/3, CA4, $\mathrm{ML}$, and right GC-DG subfields were significantly smaller in $\mathrm{PD}$ patients. Also, there were significant correlations between the MoCA score and total hippocampus volume in $\mathrm{PD}$ patients. In the follow-up groups, total bilateral hippocampal volume, bilateral CA4, ML, and GC-DG subfields, and the left CA2/3 and right presubiculum subfields were also significantly atrophied in PD patients with cognitive decline compared to patients without cognitive decline. Furthermore, there were significant correlations between the longitudinal change of neuropsychological scores and mean percent change rate of bilateral total hippocampal, GC-DG, ML, and CA4 in all PD patients.

Structural MRI imaging was regarded as a recent biomarker for neurodegeneration. To date, atrophy of the total hippocampus and hippocampal subfield is a positive biomarker for dementia found in Alzheimer's disease (AD) (25) and vascular dementias (26). Total hippocampal and presubiculum atrophy in MCI is highly predictive of the future development of dementia $(25,27)$. Many studies have found focal atrophy of the CA1 subfield in the early (predementia or even preclinical) stages of $\mathrm{AD}$ $(28,29)$. However. what the structural imaging biomarkers for cognition status in PD actually have not yet been well established. Meanwhile hippocampal atrophy has been previously reported in imaging studies of $\mathrm{PD}$ with dementia and non-demented PD (30,31). Despite this, few neuroimaging studies have separately measured specific subregions within the hippocampus in non-demented PD 
Table 2 Hippocampal subfield volumes (mm3) of participants in baseline

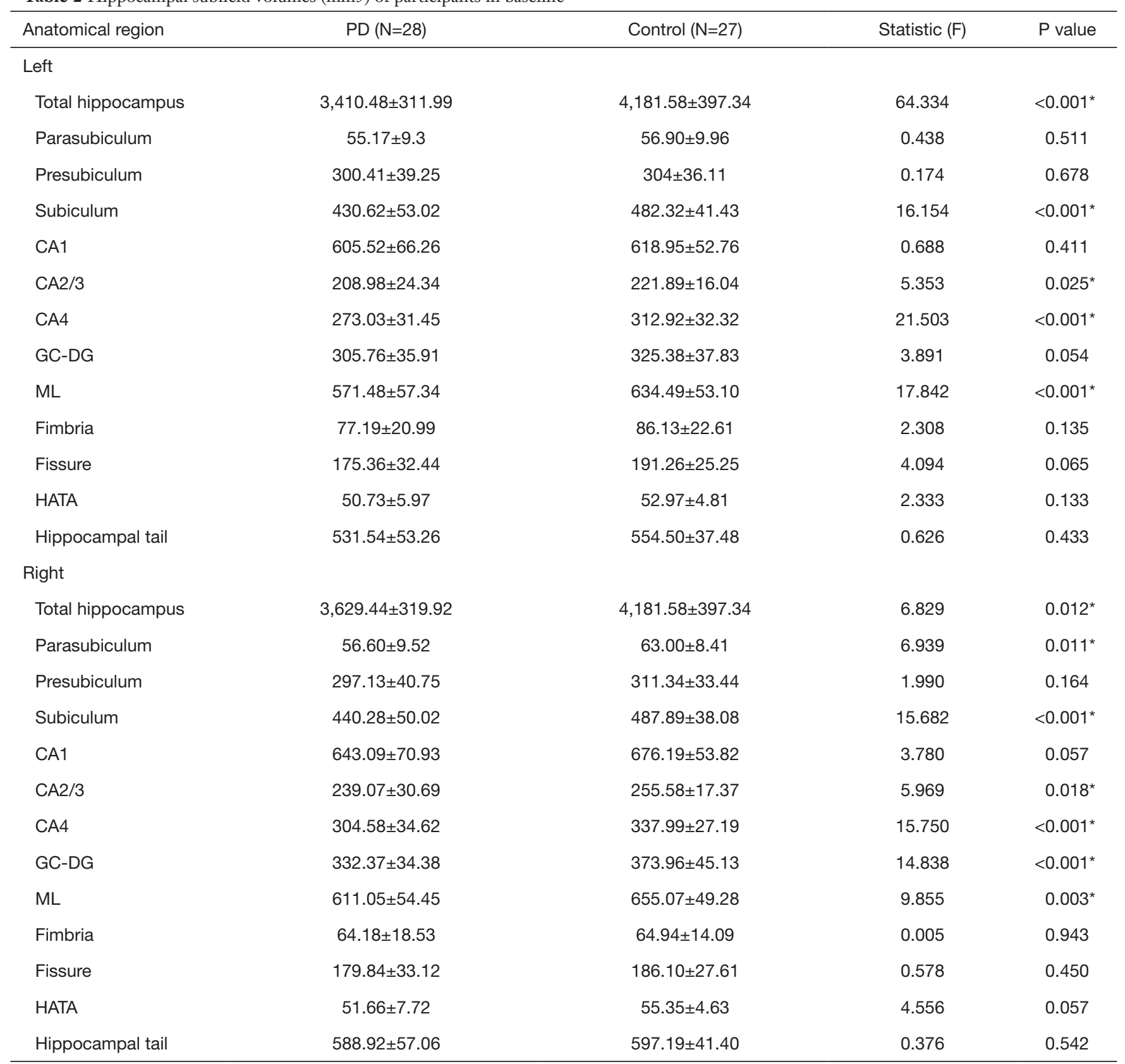

*, $P<0.05$. The $P$ value was calculate using ANCOVA, controlling for age, sex, education, and intracranial volume (ICV). CA, cornus ammonis; GC-DG, granule cell layer of dentate gyrus; M, molecular layer; HATA, hippocampus-amygdala transition area; PD, Parkinson's disease.

longitudinally.

Our study showed significant volume loss in the hippocampal CA2/3, CA4, and DG subfields in PD patients. Several mechanisms underlying volume loss of the subfields in PD have been proposed. Histopathological studies have indicated that Lewy bodies and Lewy neurites are primarily observed in the CA2 and CA3 subfields, and pathological evidence of Lewy neurite burden and an Alzheimer's disease-like pattern of hippocampal atrophy have been observed in PD patients with MCI $(11,32)$. The DG, like the CA2/3 subfield, shows a-synuclein presynaptic axon pathology in postmortem PD brains that could impair 



Figure 1 Differences between baseline groups in hippocampal subfields volumes and correlations between total hippocampus volumes and MoCA score in PD patients. CA, cornus ammonis; GC-DG, granule cell layer of dentate gyrus; ML, molecular layer; HATA, hippo-campusamygdala transition area; graphic presenting data distribution with average means and standard errors. * indicates significant results.

Table 3 Demographic information and clinical characteristics in PD patients at baseline

\begin{tabular}{|c|c|c|c|c|}
\hline Variable & PD without cognitive decline $(\mathrm{N}=15)$ & PD with cognitive decline $(\mathrm{N}=13)$ & Statistic $\left(\chi^{2 / t / Z)}\right.$ & $P$ value \\
\hline Gender (male/female) ${ }^{\mathrm{a}}$ & $6 / 9$ & $5 / 8$ & - & 1.000 \\
\hline Age $(\text { year })^{b}$ & $57.60 \pm 11.10$ & $61.23 \pm 11.27$ & -0.857 & 0.399 \\
\hline Education (year) ${ }^{\mathrm{b}}$ & $8.53 \pm 3.68$ & $9.53 \pm 4.99$ & -0.612 & 0.546 \\
\hline MMSE $^{\mathrm{c}}$ & $27.66 \pm 2.41$ & $28.92 \pm 1.49$ & -1.153 & 0.249 \\
\hline Disease duration (year) $^{\mathrm{C}}$ & $4.75 \pm 3.09$ & $4.06 \pm 2.22$ & -0.741 & 0.459 \\
\hline UPDRS-III score ${ }^{\mathrm{b}}$ & $15.33 \pm 4.45$ & $16.78 \pm 6.77$ & -0.677 & 0.505 \\
\hline Levodopa dosage $(\mathrm{mg} / \text { day })^{\mathrm{b}}$ & $308.82 \pm 59.12$ & $314.49 \pm 52.91$ & -0.266 & 0.793 \\
\hline
\end{tabular}

${ }^{a}$, the $\mathrm{P}$ value for gender distribution in the two groups was calculated using Fisher exact test. ${ }^{\mathrm{b}}$, the $\mathrm{P}$ value was calculate using two-tail two-sample $t$-test. ' , the $\mathrm{P}$ value was calculate using two-tail Mann-Whitney U test. MMSE, Mini Mental State Examination; MoCA, Montreal Cognitive Assessment; PD, Parkinson's disease. 
Table 4 Neuropsychological test scores at baseline and follow-up in PD patients

\begin{tabular}{|c|c|c|c|c|c|c|}
\hline & \multicolumn{2}{|c|}{ PD without cognitive decline $(\mathrm{N}=15)$} & \multicolumn{2}{|c|}{ PD with cognitive decline $(\mathrm{N}=13)$} & $\mathrm{F}$ & P value \\
\hline MMSE & $27.66 \pm 2.41$ & $28.33 \pm 1.75$ & $28.92 \pm 1.49$ & $27.30 \pm 2.05$ & 7.690 & $<0.001$ \\
\hline MoCA & $26.00 \pm 1.19$ & $26.08 \pm 1.32$ & $26.76 \pm 1.73$ & $23.92 \pm 3.42$ & 15.832 & 0.001 \\
\hline
\end{tabular}

${ }^{a}$, values in bold indicate significant results from repeated-measures ANOVA by mixed-effects model. MMSE, Mini Mental State Examination; MoCA, Montreal Cognitive Assessment; PD, Parkinson's disease.

Table 5 Neuropsychological test scores at baseline and follow-up in PD patients

\begin{tabular}{cccccc}
\hline & Group & Baseline & Follow-up & Z-value Wilcoxon test (baseline vs. follow-up) & P value \\
\hline MMSE & PD without CD & $27.66 \pm 2.41$ & $28.33 \pm 1.75$ & -1.841 & 0.066 \\
& PD with CD & $28.92 \pm 1.49$ & $27.30 \pm 2.05$ & -2.980 & $0.003^{*}$ \\
\multirow{2}{*}{ MoCA } & PD without CD & $26.00 \pm 1.19$ & $26.08 \pm 1.32$ & 2.588 & $0.010^{*}$ \\
& PD with CD & $26.76 \pm 1.73$ & $23.92 \pm 3.42$ & -2.449 & $0.014^{*}$ \\
\hline
\end{tabular}

${ }^{*}, \mathrm{P}<0.05$, MMSE, Mini Mental State Examination; MoCA, Montreal Cognitive Assessment; CD, cognitive decline; PD, Parkinson's disease.

normal synaptic release, produce synaptic dysfunction, and eventually degenerate presynaptic terminals (33). Previous studies also found the atrophy of CA2/3 and CA4-DG in PD using 1.5 T MRI relative to matched healthy control individuals, which was highly consistent with the current study (34).

Moreover, the hippocampal CA2/3, CA4, and DG subfields appeared sensitive in PD with cognitive decline longitudinally. The increasing volume loss over time could relate to the progression of PD. However, volume loss of the combined CA2, CA3, and DG area in $\mathrm{AD}$ has only been reported in a few in vivo MRI studies $(35,36)$, and most studies highlighted a focal atrophy of the CA1 subfield in the early stages of AD (29), which was found to correlate with the density of NFT (28). Thus, these results indicate different hippocampal regional vulnerabilities in different neurodegenerative diseases. Churchyard and Lees suggested that the Lewy neurite formation process in CA2/3 and DG produces hippocampal dysfunction and impairs cognitive function, while the pathological change in the CA2/3 and DG was associated with the severity of dementia (11). In view of the high proportion of dementia in PD patients (37), we speculate that the atrophy of CA2/3, CA4, and DG subfields longitudinally could represent an indicator for the conversion from non-demented to dementia in PD patients.

Furthermore, our study showed significant correlations between the longitudinal change of neuropsychological scores and mean percent change rate of bilateral total hippocampal, GC-DG, ML, and CA4 in all PD patients, which confirms our suspicions that CA2/3 and CA4-DG volume loss is related to cognitive decline in $\mathrm{PD}$ patients. Previous studies in healthy subjects have shown that these different hippocampal subfields make distinct contributions to the stages of memory processing $(38,39)$. The CA4 and DG sectors are close to the CA3 subfield spatially, and pathological evidence has demonstrated the presence of presynaptic $\alpha$-synuclein burden in the DG in postmortem PD brains. The pathological change could disrupt synaptic function and, in turn, degenerate presynaptic terminals (33). Previous studies have suggested that general cognitive ability is positively associated with CA4 volumes and ML development (20); furthermore, CA4/DG volume was found to predict concurrent associative memory performance (40). Our findings of a correlation between global cognitive status and GC-DG, ML, and CA4 volumes in $\mathrm{PD}$ patient samples are consistent with these studies, and confirm the crucial role of the hippocampus (41) and specific subfields $(38,39)$ in cognitive dysfunction and disease progression in PD.

The assessment of global cognitive status in the PD patients was highly consistent with previous studies $(18,42)$. However, the current study solely focused on cognitive dysfunction and did not assess psychotic symptoms, which limited our ability to covary the results for the presence 
Table 6 Hippocampal subfield volumes $\left(\mathrm{mm}^{3}\right)$ of participants in the longitudinal study

\begin{tabular}{|c|c|c|c|c|c|c|}
\hline Anatomical region & \multicolumn{2}{|c|}{ PD without cognitive decline $(\mathrm{N}=15)$} & \multicolumn{2}{|c|}{ PD with cognitive decline $(\mathrm{N}=13)$} & $\mathrm{F}$ & $P$ value \\
\hline \multicolumn{7}{|l|}{ Left } \\
\hline Total hippocampus & $3,440.09 \pm 381.90$ & $4,001.72 \pm 410.02$ & $3,376.32 \pm 215.63$ & $3,824.42 \pm 348.99$ & 6.590 & $0.016^{*}$ \\
\hline Parasubiculum & $53.07 \pm 10.67$ & $53.76 \pm 10.21$ & $57.60 \pm 7.17$ & $57.42 \pm 8.66$ & 0.453 & 0.507 \\
\hline Subiculum & $435.33 \pm 61.71$ & $446.88 \pm 77.68$ & $425.18 \pm 42.68$ & $418.68 \pm 42.36$ & 1.424 & 0.244 \\
\hline CA1 & $607.36 \pm 75.45$ & $619.80 \pm 84.68$ & $603.39 \pm 56.82$ & $588.18 \pm 53.54$ & 0.480 & 0.494 \\
\hline $\mathrm{CA} 2 / 3$ & $213.19 \pm 24.78$ & $213.92 \pm 27.17$ & $204.12 \pm 23.85$ & $196.23 \pm 22.87$ & 11.782 & $0.002^{*}$ \\
\hline CA4 & $281.44 \pm 38.57$ & $285.21 \pm 38.72$ & $263.33 \pm 17.33$ & $255.78 \pm 16.41$ & 8.704 & $0.007^{\star}$ \\
\hline Fimbria & $78.48 \pm 23.68$ & $80.62 \pm 30.03$ & $75.70 \pm 18.24$ & $71.47 \pm 18.88$ & 0.998 & 0.327 \\
\hline Fissure & $174.39 \pm 35.57$ & $174.07 \pm 27.17$ & $176.46 \pm 29.82$ & $185.31 \pm 35.90$ & 1.736 & 0.199 \\
\hline HATA & $50.68 \pm 6.98$ & $53.52 \pm 11.96$ & $50.79 \pm 4.83$ & $50.16 \pm 4.48$ & 1.261 & 0.272 \\
\hline Hippocampal tail & $525.18 \pm 48.02$ & $518.10 \pm 46.09$ & $538.87 \pm 59.86$ & $512.44 \pm 44.63$ & 0.048 & 0.828 \\
\hline \multicolumn{7}{|l|}{ Right } \\
\hline Total hippocampus & $3,531.29 \pm 361.07$ & $3,531.29 \pm 369.00$ & $3,742.70 \pm 228.56$ & $3,618.78 \pm 232.40$ & 10.034 & $0.004^{*}$ \\
\hline Parasubiculum & $53.16 \pm 7.35$ & $52.68 \pm 8.2$ & $60.57 \pm 10.44$ & $59.54 \pm 10.74$ & 0.668 & 0.421 \\
\hline Presubiculum & $288.61 \pm 46.02$ & $292.37 \pm 45.94$ & $306.97 \pm 32.71$ & $292.38 \pm 33.37$ & 11.637 & $0.002^{*}$ \\
\hline GC-DG & $322.48 \pm 34.72$ & $322.33 \pm 33.24$ & $343.78 \pm 31.48$ & $331.58 \pm 29.93$ & 6.659 & $0.016^{*}$ \\
\hline ML & $594.16 \pm 58.26$ & $594.54 \pm 62.92$ & $630.54 \pm 44.09$ & $608.68 \pm 45.66$ & 6.950 & $0.014^{*}$ \\
\hline Fimbria & $62.17 \pm 20.53$ & $62.48 \pm 18.69$ & $67.45 \pm 16.25$ & $65.20 \pm 15.38$ & 1.124 & 0.299 \\
\hline Fissure & $169.18 \pm 29.54$ & $181.02 \pm 32.20$ & $192.13 \pm 33.84$ & $190.86 \pm 31.60$ & 4.391 & 0.056 \\
\hline HATA & $50.34 \pm 6.94$ & $49.42 \pm 7.3$ & $53.19 \pm 8.56$ & $52.32 \pm 7.76$ & 0.002 & 0.969 \\
\hline Hippocampal tail & $590.94 \pm 61.67$ & $582.03 \pm 62.44$ & $586.59 \pm 53.66$ & $560.83 \pm 45.23$ & 0.363 & 0.552 \\
\hline
\end{tabular}

${ }^{*}, \mathrm{P}<0.05 .{ }^{a}$, values in bold indicate significant results from repeated-measures ANOVA by mixed-effects model (group $\times$ time interaction) in comparison of hippocampal subfield volumes with age as a covariate. CA, cornus ammoni; GC-DG, granule cell layer of dentate gyrus; ML, molecular layer; HATA, hippocampus-amygdala transition area; PD, Parkinson's disease. 

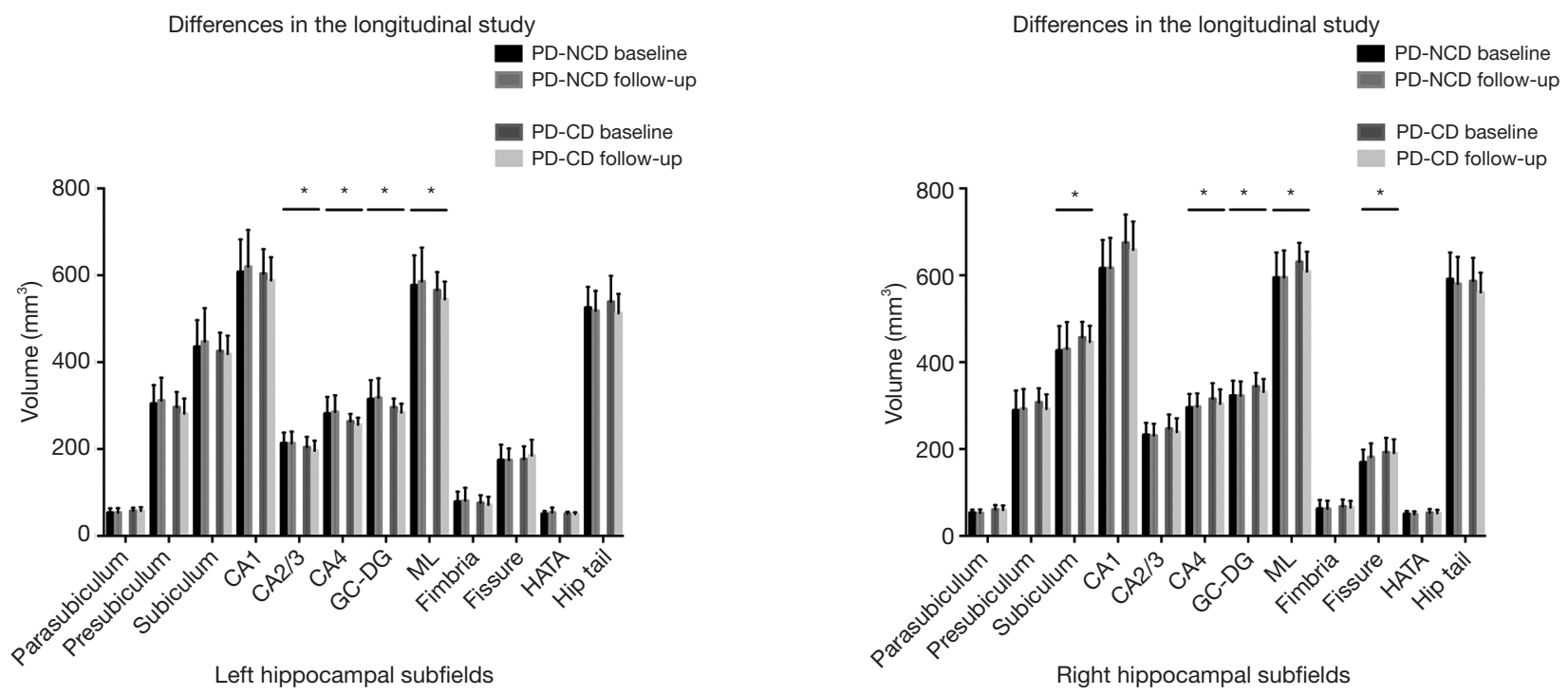

Figure 2 Differences between longitudinal groups in hippocampal subfield volumes. PD-NCD, PD without cognitive decline group; PDCD, PD with cognitive decline group; CA, cornus ammonis; GC-DG, granule cell layer of dentate gyrus; ML, molecular layer; HATA, hippocampus-amygdala transition area; graphic presenting data distribution with average means and standard errors. *, indicates significant results.

of psychotic symptoms. There is increasing evidence for a strong relationship between psychotic symptoms and hippocampal atrophy $(43,44)$, as shown in a longitudinal study on psychosis, and psychosis in patients with PD has also been recently related to a smaller hippocampal volume (45). Given these facts, future studies should focus on the interactions between cognitive symptoms and psychotic symptoms, along with their influence on the hippocampus and specific subfields.

There are several limitations to this study. First, our study had a small sample size, which limited our statistical power and the ability to reach significance in several of the analyses. In addition, healthy volunteers were not enrolled over time in the study, which weakens the rigor and persuasiveness of the study. Furthermore, only a global measure of cognition was used. The subjects did not receive a comprehensive neuropsychological battery, which limited our ability to assess more specific associations between structural alterations and cognitive decline. Thus, future studies need to enroll larger samples of patients and healthy volunteers longitudinally, with more detailed assessment of cognitive domains, which could increase the ability to draw specific conclusions about cognitive decline longitudinally in PD as compared to normal aging. Third, most of the PD patients were under dopaminergic medications, and the effects of dopaminergic medications were not excluded.
Moreover, there were methodological issues, one of which was that the MRI resolution restricted hippocampal subfield segmentation. However, the latest versions of FreeSurfer was used to improve the sensitivity to identify group differences, and this version has consistency with the results of the manual segmentation technique (21). Meanwhile, the signal-to-noise characteristics at 3 Tesla in the current study were improved over 1.5 Tesla with the routine T1-weighted scans (46).

\section{Conclusions}

Neuroimaging offers great promise for the study of disease pathogenesis and the evolution of non-motor syndromes, particularly in studying brain structure changes in PD. We explored the pattern of reductions in the volume of the total hippocampus and of specific hippocampal subfields in PD patients. In particular, we report longitudinally decreased bilateral CA2/3, CA4, and DG subfield volume in PD with cognitive decline. Furthermore, these findings support the crucial role of the hippocampus in the cognition of PD patients and should contribute to the future study of cognitive symptoms in PD patients. In summary, our findings corroborate neuropathological findings and add novel information about the involvement of the hippocampus in the cognitive dysfunction of $\mathrm{PD}$. 

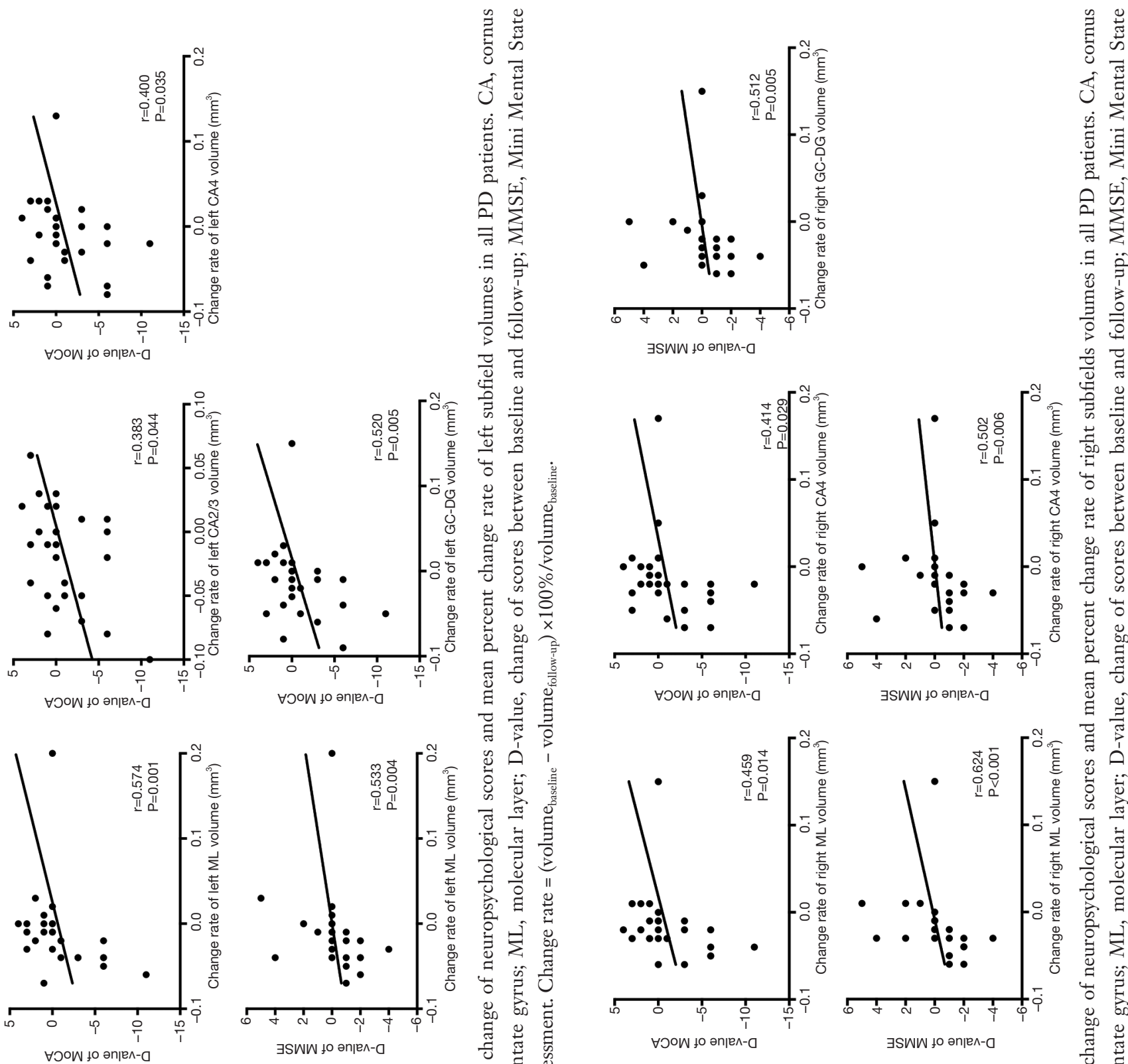

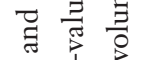

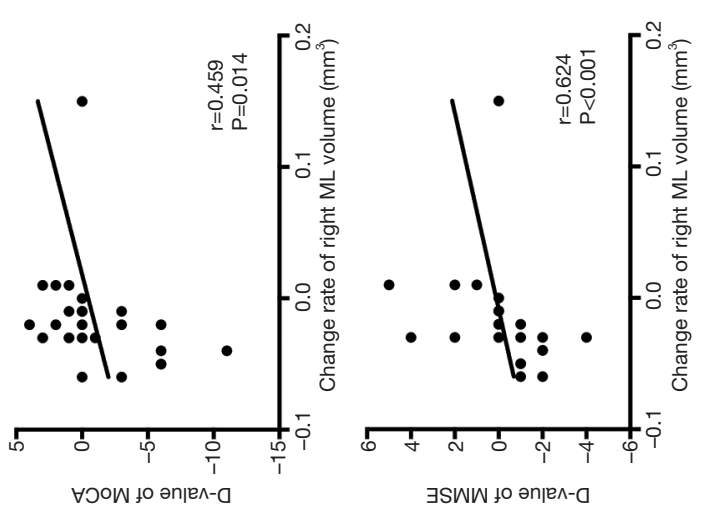


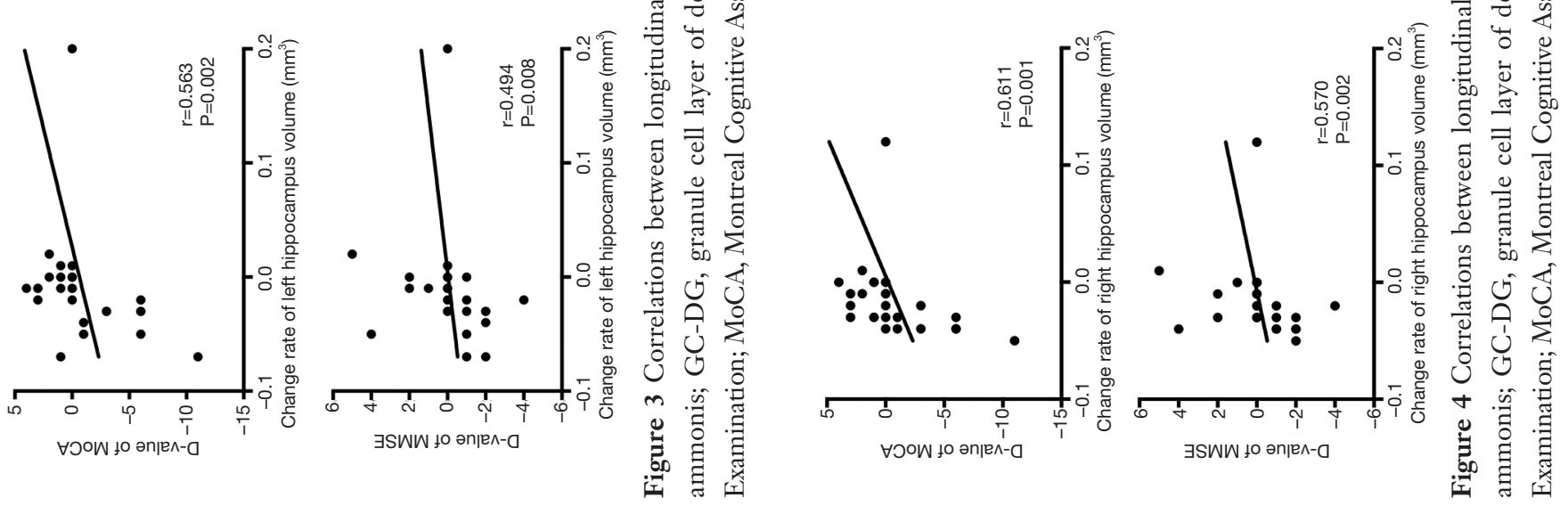


\section{Acknowledgments}

Funding: This work was supported by the National Nature Science Foundation of China (No. 81701664) and the Technology Innovation Program in Southwest Hospital (SWH2016JCYB-30).

\section{Footnote}

Conflicts of Interest: The authors have no conflicts of interest to declare.

Ethical Statement: The present study was approved by the Ethics Committee of Southwest Hospital, and written informed consent to participate in the study was obtained from all subjects.

\section{References}

1. Cholerton BA, Zabetian CP, Wan JY, Montine TJ, Quinn JF, Mata IF, Chung KA, Peterson A, Espay AJ, Revilla FJ, Devoto J, Watson GS, Hu SC, Leverenz JB, Edwards KL. Evaluation of mild cognitive impairment subtypes in Parkinson's disease. Mov Disord 2014;29:756-64.

2. Williams-Gray CH, Evans JR, Goris A, Foltynie T, Ban M, Robbins TW, Brayne C, Kolachana BS, Weinberger DR, Sawcer SJ, Barker RA. The distinct cognitive syndromes of Parkinson's disease: 5 year follow-up of the CamPaIGN cohort. Brain 2009;132:2958-69.

3. Benito-Leon J, Louis ED, Posada IJ, Sanchez-Ferro A, Trincado R, Villarejo A, Mitchell AJ, Bermejo-Pareja F. Population-based case-control study of cognitive function in early Parkinson's disease (NEDICES). J Neurol Sci 2011;310:176-82.

4. Aarsland D, Bronnick K, Larsen JP, Tysnes OB, Alves G, Norwegian ParkWest Study G. Cognitive impairment in incident, untreated Parkinson disease: the Norwegian ParkWest study. Neurology 2009;72:1121-6.

5. Muslimovic D, Post B, Speelman JD, De Haan RJ, Schmand B. Cognitive decline in Parkinson's disease: a prospective longitudinal study. J Int Neuropsychol Soc 2009; 15:426-37.

6. Williams-Gray CH, Foltynie T, Brayne CE, Robbins TW, Barker RA. Evolution of cognitive dysfunction in an incident Parkinson's disease cohort. Brain 2007;130:1787-98.

7. Carlesimo GA, Piras F, Assogna F, Pontieri FE, Caltagirone C, Spalletta G. Hippocampal abnormalities and memory deficits in Parkinson disease: a multimodal imaging study. Neurology 2012;78:1939-45.

8. Junque C, Ramirez-Ruiz B, Tolosa E, Summerfield C, Marti MJ, Pastor P, Gomez-Anson B, Mercader JM. Amygdalar and hippocampal MRI volumetric reductions in Parkinson's disease with dementia. Mov Disord 2005;20:540-4.

9. Tam CW, Burton EJ, McKeith IG, Burn DJ, O'Brien JT. Temporal lobe atrophy on MRI in Parkinson disease with dementia: a comparison with Alzheimer disease and dementia with Lewy bodies. Neurology 2005;64:861-5.

10. Braak H, Del Tredici K, Rub U, de Vos RA, Jansen Steur EN, Braak E. Staging of brain pathology related to sporadic Parkinson's disease. Neurobiol Aging 2003;24:197-211.

11. Churchyard A, Lees A. The relationship between dementia and direct involvement of the hippocampus and amygdala in Parkinson's disease. Neurology 1997;49:1570-6.

12. Kalaitzakis ME, Christian LM, Moran LB, Graeber MB, Pearce RK, Gentleman SM. Dementia and visual hallucinations associated with limbic pathology in Parkinson's disease. Parkinsonism Relat Disord 2009;15:196-204.

13. Oldfield RC. The assessment and analysis of handedness: the Edinburgh inventory. Neuropsychologia 1971;9:97-113.

14. Daniel SE, Lees AJ. Parkinson's Disease Society Brain Bank, London: overview and research. J Neural Transm Suppl 1993;39:165-72.

15. Dubois B, Burn D, Goetz C, Aarsland D, Brown RG, Broe GA, Dickson D, Duyckaerts C, Cummings J, Gauthier S. Diagnostic procedures for Parkinson's disease dementia: recommendations from the movement disorder society task force. Mov Disord 2007;22:2314-24.

16. Fahn S, Elton R. Unified rating scale for Parkinson's disease. Recent developments in Parkinson's disease. Florham Park, New York: Macmillan, 1987:153-63.

17. Hoehn MM, Yahr MD. Parkinsonism: onset, progression and mortality. Neurology 1967;17:427-42.

18. Hu MT, Szewczyk-Krolikowski K, Tomlinson P, Nithi K, Rolinski M, Murray C, Talbot K, Ebmeier KP, Mackay CE, Ben-Shlomo Y. Predictors of cognitive impairment in an early stage Parkinson's disease cohort. Mov Disord 2014;29:351-9.

19. Kandiah N, Zhang A, Cenina AR, Au WL, Nadkarni N, Tan LC. Montreal Cognitive Assessment for the screening and prediction of cognitive decline in early Parkinson's disease. Parkinsonism Relat Disord 2014;20:1145-8.

20. Tamnes CK, Bos MGN, van de Kamp FC, Peters S, Crone 
EA. Longitudinal development of hippocampal subregions from childhood to adulthood. Dev Cogn Neurosci 2018;30:212-22.

21. Iglesias JE, Leemput KV, Augustinack J, Insausti R, Fischl B, Reuter M. Bayesian longitudinal segmentation of hippocampal substructures in brain MRI using subjectspecific atlases. Neuroimage 2016;141:542-55.

22. Iglesias JE, Augustinack JC, Nguyen K, Player CM, Player A, Wright M, Roy N, Frosch MP, Mckee AC, Wald LL. A computational atlas of the hippocampal formation using ex vivo, ultra-high resolution MRI: Application to adaptive segmentation of in vivo MRI. Neuroimage 2015;115:117-37.

23. Du AT, Schuff N, Amend D, Laakso MP, Hsu YY, Jagust WJ, Yaffe K, Kramer JH, Reed B, Norman D, Chui HC, Weiner MW. Magnetic resonance imaging of the entorhinal cortex and hippocampus in mild cognitive impairment and Alzheimer's disease. J Neurol Neurosurg Psychiatry 2001;71:441-7.

24. Ibarretxe-Bilbao N, Junque C, Segura B, Baggio HC, Marti MJ, Valldeoriola F, Bargallo N, Tolosa E. Progression of cortical thinning in early Parkinson's disease. Mov Disord 2012;27:1746-53.

25. Khan W, Westman E, Jones N, Wahlund LO, Mecocci P, Vellas B, Tsolaki M, Kłoszewska I, Soininen H, Spenger C. Automated hippocampal subfield measures as predictors of conversion from mild cognitive impairment to Alzheimer's disease in two independent cohorts. Brain Topogr 2015;28:746-59.

26. Xu Y, Valentino DJ, Scher AI, Dinov I, White LR, Thompson PM, Launer LJ, Toga AW. Age effects on hippocampal structural changes in old men: the HAAS. Neuroimage 2008;40:1003-15.

27. Apostolova LG, Thompson PM, Green AE, Hwang KS, Zoumalan C, Jack CR Jr, Harvey DJ, Petersen RC, Thal LJ, Aisen PS, Toga AW, Cummings JL, Decarli CS. 3D comparison of low, intermediate, and advanced hippocampal atrophy in MCI. Hum Brain Mapp 2010;31:786-97.

28. Small SA, Schobel SA, Buxton RB, Witter MP, Barnes CA. A pathophysiological framework of hippocampal dysfunction in ageing and disease. Nat Rev Neurosci 2011;12:585-601.

29. Flores RD, Joie RL, Chételat G. Structural imaging of hippocampal subfields in healthy aging and Alzheimer's disease. Neuroscience 2015;309:29-50.

30. Apostolova L, Alves G, Hwang KS, Babakchanian S, Bronnick KS, Larsen JP, Thompson PM, Chou YY, Tysnes OB, Vefring HK, Beyer MK. Hippocampal and ventricular changes in Parkinson's disease mild cognitive impairment. Neurobiol Aging 2012;33:2113-24.

31. Bouchard TP, Malykhin N, Martin WR, Hanstock CC, Emery DJ, Fisher NJ, Camicioli RM. Age and dementiaassociated atrophy predominates in the hippocampal head and amygdala in Parkinson's disease. Neurobiol Aging 2008;29:1027-39.

32. Mattila PM, Rinne JO, Helenius H, Roytta M. Neuritic degeneration in the hippocampus and amygdala in Parkinson's disease in relation to Alzheimer pathology. Acta Neuropathol 1999;98:157-64.

33. Galvin JE, Uryu K, Lee VM, Trojanowski JQ. Axon pathology in Parkinson's disease and Lewy body dementia hippocampus contains alpha-, beta-, and gamma-synuclein. Proc Natl Acad Sci U S A 1999;96:13450-5.

34. Pereira JB, Junque C, Bartres-Faz D, Ramirez-Ruiz B, Marti MJ, Tolosa E. Regional vulnerability of hippocampal subfields and memory deficits in Parkinson's disease. Hippocampus 2013;23:720-8.

35. Joie RL, Perrotin A, Sayette VDL, Egret S, Doeuvre L, Belliard S, Eustache F, Desgranges B, Chételat G. Hippocampal subfield volumetry in mild cognitive impairment, Alzheimer's disease and semantic dementia. Neuroimage Clin 2013;3:155-62.

36. Qiu A, Fennema-Notestine C, Dale AM, Miller MI. Regional shape abnormalities in mild cognitive impairment and Alzheimer's disease. Neuroimage 2009;45:656-61.

37. Beyer MK, Bronnick KS, Hwang KS, Bergsland N, Tysnes OB, Larsen JP, Thompson PM, Somme JH, Apostolova LG. Verbal memory is associated with structural hippocampal changes in newly diagnosed Parkinson's disease. J Neurol Neurosurg Psychiatry 2013;84:23-8.

38. Eldridge LL, Engel SA, Zeineh MM, Bookheimer SY, Knowlton BJ. A dissociation of encoding and retrieval processes in the human hippocampus. J Neurosci 2005;25:3280-6.

39. Carr VA, Viskontas IV, Engel SA, Knowlton BJ. Neural activity in the hippocampus and perirhinal cortex during encoding is associated with the durability of episodic memory. J Cogn Neurosci 2010;22:2652-62.

40. Bender AR, Daugherty AM, Naftali R. Vascular risk moderates associations between hippocampal subfield volumes and memory. J Cogn Neurosci 2013;25:1851-62.

41. Hu MT, White SJ, Chaudhuri KR, Morris RG, Bydder GM, Brooks DJ. Correlating rates of cerebral atrophy in Parkinson's disease with measures of cognitive decline. J Neural Transm (Vienna) 2001;108:571-80.

42. Bugalho P, Viana-Baptista M. Predictors of cognitive 
decline in the early stages of Parkinson's disease: a brief cognitive assessment longitudinal study. Parkinsons Dis 2013;2013:912037.

43. Lieberman JA, Girgis RR, Brucato G, Moore H, Provenzano F, Kegeles L, Javitt D, Kantrowitz J, Wall MM, Corcoran CM. Hippocampal dysfunction in the pathophysiology of schizophrenia: a selective review and hypothesis for early detection and intervention. Mol Psychiatry 2018;23:1764-72.

44. Ho NF, Iglesias JE, Sum MY, Kuswanto CN, Sitoh YY, De SJ, Hong Z, Fischl B, Roffman JL, Zhou J. Progression

Cite this article as: $\mathrm{Xu} \mathrm{R}, \mathrm{Hu} \mathrm{X}$, Jiang $\mathrm{X}$, Zhang $\mathrm{Y}$, Wang J, Zeng X. Longitudinal volume changes of hippocampal subfields and cognitive decline in Parkinson's disease. Quant Imaging Med Surg 2020;10(1):220-232. doi: 10.21037/qims.2019.10.17 from selective to general involvement of hippocampal subfields in schizophrenia. Mol Psychiatry 2017;22:142-52.

45. Lenka A, Ingalhalikar M, Shah A, Saini J, Arumugham SS, Hegde S, George L, Reddy V, Reddy YCJ, Yadav R. Hippocampal subfield atrophy in patients with Parkinson's disease and psychosis. J Neural Transm (Vienna) 2018;125:1361-72.

46. Jeukens CR, Vlooswijk MC, Majoie HJ, de Krom MC, Aldenkamp AP, Hofman PA, Jansen JF, Backes WH. Hippocampal MRI volumetry at 3 Tesla: reliability and practical guidance. Invest Radiol 2009;44:509-17. 